\title{
On the Impact of Zucchini Yellow and Watermelon Mosaic Viruses on the Production Rhythms of Transgenic ZW-20 and Nontransgenic Squash (Cucurbita pepo L.)
}

\author{
Ferdinand E. Klas ${ }^{1 *}$ \\ ${ }^{1}$ Anton de Kom University of Suriname, Faculty of Technology, University Campus Leysweg, \\ Building XVII, POB 12292, Paramaribo, Suriname.
}

Author's contribution

Within the USDA Biotechnology Risk Assessment Program (No. 95-33120-1878) the author conducted experiments to study the impact of transgenic squash on the spread dynamics of cucurbit viruses. In view of his mathematical statistical expertise the author was free to design and adapt field and laboratory experiments to achieve these goals. Data collection and statistical analysis of collected data were fully performed by the author. All versions (first draft and revised versions) of the manuscript were written by the author.

Research Article

Received $8^{\text {th }}$ March 2012

Accepted 17 $7^{\text {th }}$ June 2012

Online Ready $11^{\text {th }}$ July 2012

\section{ABSTRACT}

Throughout the growing season the numbers of harvested young fruits that reached marketable size fluctuated per unit of time, depicting the production rhythm of the crop. Most studies on the impact of disease on a crop pertain to the extent of yield reductions caused, and not to perturbation or annihilation of the production rhythm.

On the Crittenden Farm of the New York State Agricultural Experiment Station in Geneva, NY, field trials were conducted to study the impact of cucurbit viruses Zucchini yellow mosaic (ZYMV) and Watermelon mosaic (WMV) on production rhythms of transgenic ZW$20(\mathrm{H}$ and $\mathrm{B})$ and nontransgenic cv Pavo squash. Both, $\mathrm{ZW}-20 \mathrm{H}$ and $\mathrm{ZW}-20 \mathrm{~B}$ were genetically engineered from cV Pavo and expressed virus-resistance evoked by coat protein genes of ZYMV and WMV. The nontransgenic cV. Pavo control did not contain virus resistance and was fully susceptible to ZYMV and WMV. Production rhythms were depicted by time series of successive average numbers of small fruits of marketable size, harvested at 2-3 day intervals between 31 and 49 days post planting (dpp). For both, 
virus-infected and virus-free transgenic $\mathrm{ZW}-20(\mathrm{H}$ and $\mathrm{B}$ ), as well as for virusfree nontransgenic cv Pavo the trend was manifest that, at each harvest the number of fruits was higher than at the previous one; indicating the existence of autocorrelation between harvest rounds of these genotypes. In contrast, this trend of increasing numbers of fruits could not be identified with virus-infected nontransgenic cv Pavo. Consistent with these trends, Von Neumann's $Q$ confirmed the occurrence of significant autocorrelations between harvest rounds of ZW-20 (both, virusfree and infected) and of virus free nontransgenic cv Pavo. In contrast, Von Neumann's Q did not detect autocorrelation between harvest rounds of virus-infected nontransgenic cv Pavo. These results reveal that autocorrelation between harvest rounds was retained in transgenic ZW-20 squash, irrespective if they were virus-infected or not, but was lost in infected virus-susceptible nontransgenic cv Pavo crops. The outcomes of this study sustain the hypothesis that ZYMV and WMV have no impact on the production rhythms of virus-resistant transgenic ZW-20 squash, but they do alter those of nontransgenic cv Pavo.

Keywords: Phenotypic plasticity; fruit removal; autocorrelation; von Neumann.

\section{INTRODUCTION}

\subsection{General Introduction}

Plants accumulate resources such as water, mineral nutrients and photosynthate and allocate them to growth, maintenance and defense (Bazzaz, 1997). Because most plants are hermaphrodites (Yampolski and Yampolski, 1922), the resources allocated to reproduction must be further divided in male (pollen production) and female (fruit and seed production) functions (Charlesworth and Charlesworth, 1982). A number of studies that have manipulated either resource availability (resource enrichment and deprivation studies) or demand (such as staminate flower production and/or fruit initiation) have demonstrated trade-offs between plant functions, such as growth versus reproduction, and defense versus reproduction (Schlichting and Pigliucci, 1998; Agrawal, 1999; Cipolini, 1999). For all these trade-offs the theory of phenotypic plasticity should hold. This theory predicts that natural selection should favor individuals with the capacity to change patterns of resource allocation to match the new conditions in such a way that fitness is maintained (Dudley and Schmitt, 1995; Schmitt et al., 1995, 1999; Agrawal, 1999; Cipolini and Schultz, 1999).

Cucurbits, like many crops are raised as annuals. Resources conserved in an annual crop from the current reproduction cannot be held over until the following reproductive session, but they can be reallocated to increase fitness later in the same reproductive session. In a number of crops it has been shown also that developing fruits enjoy priority in terms of their demand on assimilates from adjacent leaves and that fruit development occurs at the expense of additional vegetative growth (Delesalle and Mooreside, 1995). This process makes fruits very strong sinks of carbon and mineral nutrients (Wardlaw, 1990). Therefore, by removing immature small fruits at the earliest stages of their development, the plant should enjoy a surplus of resources as compared to plants with maturing fruits. These extra resources that come free, according to sex allocation and phenotypic plasticity theory, should then become available for other functions like flower and pollen production, growth and defense (Charnov, 1982; Watson and Casper, 1984). 


\subsection{Specific Introduction}

El-Keblawy and Louvett-Doust (1996) found that removal of squash fruits favored vegetative growth in terms of increasing numbers of nodes, which stimulated the production of additional leaves, stem and flowers compared with control individuals (from which no fruits were removed). This means that resources that became available in treated plants could be reallocated to flower and fruit production within the response time. Similarly was also detected for Cucurbita pepo L. spp texana, when flowers were removed after bloom (Krupnick et al., 1999; Avilla-Sakar et al., 2001). This resulted in plants with twice as many branches, longer internodes and production of more nodes per day compared with untreated plants (fruits allowed to mature). With more nodes and branches (vegetative growth), the plants also produced more pistillate flowers and thus more small squash fruits (fruiting).

Altogether, from above-mentioned studies the phenomenon is manifest that removal of fruits in an early stage of their development triggers the reproductive potential of the plant to produce more fruits at each harvest. This fruiting-growth process (production rhythm) is well known to squash growers and has become common practice in squash production. Therefore, growers do not allow large fruits to remain on the plants as this reduces additional fruit set. Early fruit removal links and correlates successive fruit productions (harvests), and is therefore the basis for the existence of autocorrelation between successive harvests in a disease free crop. Consequently, in this study production rhythm is defined in terms of the existence of autocorrelation between successive harvests.

Squash genotypes, selected to conduct the experiments, consisted of two transgenic lines of ZW-20 and one nontransgenic line, cv Pavo. The ZW-20 lines were resistant, but not immune to virus infection. Firstly $\mathrm{ZW}-20 \mathrm{H}$, included plants that were symptomless or eventually developed mild localized chlorotic dots upon infection by Zucchini yellow mosaic (ZYMV) or Watermelon mosaic (WMV). These plants contained the $\mathrm{H}$ insert of the WMV coat protein (CP) gene (Tricoli et al., 1995). The second transgenic line was labeled ZW-20B and consisted of plants that developed both, mild localized chlorotic dots and blotches upon infection by ZYMV or WMV. Latter line lacked the H insert of the WMV CP gene (Tricoli et al., 1995). When infected and showing localized mild symptoms, both $Z W-20 \mathrm{H}$ and $Z \mathrm{~W}-20 \mathrm{~B}$ could react positively in ELISA. Fruits of both transgenic lines remained asymptomatic. In contrast, the nontransgenic cv Pavo line was susceptible to virus infection and developed severe systemic symptoms on both, leaves and fruits.

Immature fruits of marketable size (15-18 long) from, both, non-infected and virus-infected ZW-20 transgenic lines, on the one hand and from, both, non-infected and virus-infected non-transgenic cv Pavo, on the other hand, were harvested at 2-3 day time intervals. These features represented the fruiting - growth (production) rhythms of these crops.

Most studies on disease impact on a crop pertain to the extent of yield reductions caused (Fuchs et al., 1998), and do not address issues on perturbation or annihilation of the production rhythm. Therefore, in this work an impulse to this approach is given. In this respect the occurrence or absence of autocorrelation in time series of successive harvests of young immature fruits of marketable size from, both, virus free and virus-infected squash plants was characterized and quantified, using Von Neumann's $Q$ for autocorrelation.

The aim of this study was to test and evaluate whether the cucurbit viruses ZYMV and WMV had an impact on the production rhythms of early harvested immature fruits of virus-resistant 
transgenic ZW-20 squash and of virus-susceptible nontransgenic cv Pavo squash, respectively.

\section{MATERIALS AND METHODS}

\subsection{Methodology}

Each squash plant in a field can be considered as an object on which successive yields are measured by counting and weighing the fruits. Successive crop yields, therefore, constitute the successive sums of yields of individual plants. It is generally recognized that values in a time series of successive measurements on an object (e.g. plant, weather station), influenced by their position in space or by preceding observations are not random, but autoor serially correlated (Buishand, 1982; Wang, 2003).

Harvesting immature young fruits of marketable size $(15-18 \mathrm{~cm})$ provides us with a system in which at each successive harvest the plant has more nodes and therefore produces more fruits than at the previous harvest. This implies that yield at time $i+1$ is influenced by the previous harvest at time $i$, giving rise to autocorrelation between successive harvests.

A suitable quantity to assess the occurrence and extent of autocorrelation in a time series of measurements is the Von Neumann's auto- (serial) correlation statistic, $\mathbf{Q}$ (Von Neumann, 1941; Buishand, 1982):

$$
Q=\frac{\sum_{i=1}^{m-1}\left(x_{i+1}-x_{i}\right)^{2}}{\sum_{i=1}^{m}\left(x_{i}-\bar{x}\right)^{2}}
$$

where $x_{i} \quad(i=1,2, \ldots \ldots, m)$ represents the average numbers of fruits per plant at time $i$ and $x_{i+1}$ the average number of fruits per plant at time $i+1$. In this study $m=8$. The numerator of equation (1) refers to the sum of squared differences between neighboring observations over time, and the denominator stands for the sum of squared differences between individual observations and the mean of the time series. By dividing, both, denominator and numerator in equation (1) by $m-1$ the denominator is transformed into the sample variance, $s^{2}$ and the numerator into the expectation of the squared differences between neighboring observations (or mean squared successive difference). It can be derived (see appendix) that the numerator of equation (1) equals,

$$
\frac{\sum_{i=1}^{m-1}\left(x_{i+1}-x_{i}\right)^{2}}{m-1}=2 \sigma^{2}(1-\rho)
$$

The right hand side of equation (2) clearly shows the correlation coefficient $\rho$, which relates $Q$ to serial correlations. For increasing sample size, $m$, the sample variance, $s^{2}$, approaches the population variance $\sigma^{2}$, making $Q$ asymptotically equal to

$$
Q=2(1-\rho) \quad \text { as } m \rightarrow \infty
$$


This implies that for increasing correlation coefficient, $\rho$, Von Neumann's $Q$ tends to smaller values, justifying the establishment of a left-tail critical region to test for significance of calculated Q-values. That is, to test for autocorrelation.

Von Neumann's $Q$ is primarily designed to examine autocorrelation between neighboring observations (LagTime=1). To further explore this statistic also for near-adjacent observations, equation (1) was expanded to a function of Lag Time $=\mathrm{j}$.

$$
Q(j)=\frac{\sum_{i=1}^{m-j}\left(x_{i+j}-x_{i}\right)^{2}}{\sum_{i=1}^{m}\left(x_{i}-\bar{x}\right)^{2}}
$$

In this study Lag Time $=\mathrm{j}$ ranged from 1 to 4 . For $\mathrm{j}=1$ equation (1) appears.

\subsection{Materials}

\subsubsection{Plant material}

Transgenic croockneck summer squash line ZW-20 was developed by Agrobacterium tumefaciens-mediated transformation, using the binary vector pPRBN - ZYMV72 / WMBN22 and contains the CP genes of ZYMV and WMV (Tricoli et al., 1995) and the marker gene neomycin phoshotransferase II (NPT II). Transgenic hybrids ZW-20H and ZW-20B, resulting from crosses of transgenic plants, which were homozygous for the two CP transgenes and an untransformed parent (cv Pavo), were used in this study. The commercial conventional cultivar Pavo, which is susceptible to ZYMV and WMV, and has the same background as the two transgenic hybrids, was used as control.

All plants, transformed and non-transformed ones, were raised in the greenhouse. Transgenic ZW-20 plants underwent the NPT-II ELISA test to verify if transformation was successful. Vigorous plants in the 2- or 3-leaves stage were selected in the greenhouse and transplanted to the field plots. All plants were transplanted on the same day.

\section{$\underline{\text { 2.2.2 Field layout }}$}

Field trials were conducted at the Crittenden farm, New York State Agricultural Experiment Station, Geneva, NY. Four plots were planted: two with transgenic squash (ZW-20H and ZW-20B) and two with nontransgenic summer squash cv Pavo. The four field plots were laid out $20 \mathrm{~m}$ apart and consisted of 10 rows of 20 plants each. The between-row and within-row distances were $2 \mathrm{~m}$ and $1 \mathrm{~m}$, respectively. An environment of high infection pressure was established by surrounding each plot by a single row of nontransgenic cv Pavo, in alternating groups of four mechanically ZYMV-inoculated and four WMV- inoculated plants that served as virus donor. These cv Pavo virus donor plants were carborundum-dusted and then mechanically inoculated in the greenhouse prior to transplanting. Viruses were spread (treatments) at random to the different plants in each plot by indigenous aphid populations. Therefore, no insecticides were used in the open field. Fruits that had reached marketable size $(15-18 \mathrm{~cm})$, were harvested every $2-3$ day.

Two other plots were established to compare growth and production when fruits were left until maturity on the plant. These plots were of the same dimensions and design as the four mentioned above. One of these plots was planted with transgenic ZW-20B and the other 
one with nontransgenic cv Pavo. Fruits were harvested here at 20-25 days intervals and only three harvests were accomplished throughout the season. As this publication is particularly focused on the analysis of production rhythms, only pictures of this transgenic ZW-20B plot were used for comparison of fruit size and production with small immature fruits harvested at 2-3 days time intervals. Specific information on fruit yield of these genotypes is published in Klas et al. (2011).

\subsubsection{Disease spread}

Virus incidence was monitored by visual observation of symptoms on test and control plants every 3-4 day, starting one week after transplanting to the field. Double antibody sandwich (DAS) enzyme-linked immunosorbent assay (ELISA), using y-globulins specific to ZYMV and WMV (Fuchs et al., 1998) were performed at 14, 45 and $74 \mathrm{dpp}$ (days post planting). Samples were considered positive, if their optical density readings at $405 \mathrm{~nm}\left(\mathrm{OD}_{405} \mathrm{~nm}\right)$ were at least twice the values of healthy nontransgenic controls (Klas et al., 2006)

\subsubsection{Analysis of the production rhythm}

It is well known that removing summer squash fruits at 2-3 day intervals increases fruit production over time of squash plants. That is, every 2-3 day there are enough fruits to be harvested and delivered to the market. This feature represents the production rhythm of the squash crop.

The question to be addressed is: do the cucurbit viruses ZYMV and WMV have an impact on the production rhythms of transgenic ZW-20 and nontransgenic cv Pavo squash?

In this respect fruits of marketable size $(15-18 \mathrm{~cm}$ long and $3.75 \mathrm{~cm}$ in diameter) on each plant (both transgenic ZW-20 ( $\mathrm{B}$ and $\mathrm{H}$ ) and nontransgenic cv Pavo) were harvested and counted on $31,33,35,38,40,42,45,47$ and 49 days after planting. For each genotype plants were subdivided in four groups: three groups being symptomatic and tested (ELISA) positive for ZYMV, WMV and ZYMV+WMV (mixed infection) and one group that tested negative (healthy) for these viruses at the first and last ELISA tests on 14 and 74dpp, respectively. These four groups were named infection groups. Per genotype the average numbers of fruits per plant in each infection group were assessed for each harvest date, yielding time series of average numbers of fruits per infection group and plotted against DPP. These time series were then analyzed for autocorrelation, using Von Neumann's $Q$ (equation 1). For these analyses fruits counted on 31dpp were neglected, because these fruits were already there before the start of the 2-3 day harvest strategy; leaving $8(=9-1)$ sampling time points. The statistic $Q(j)$ (equation(4)) was used to further explore Von Neumann's $Q$ at Lag Times ranging from $j=2$ to 4 . A left-tail critical region for $Q$, at $P=0.05$ and sample size $m=8$ yielding a critical value of 0.9825 (Following Owen, 1962), was maintained.

\section{RESULTS}

\subsection{Symptomatology and Virus Impact on Fruit Quality and Production}

Leaves of both transgenic ZW-20 were fully expanded and symptomless, though some of these plants showed mild symptoms consisting of some localized chlorotic dots and blotches as is seen in panel A of Fig. 1. On the other hand infected nontransgenic plants exhibited 
severe systemic foliar symptoms, including mosaic, deformation and shoestringing (Fig. 1 panel B). Fruits of infected transgenic ZW-20B were all symptomless and marketable (Fig. 1 panel $\mathrm{C}$ ). Fruits of infected nontransgenic cv Pavo, on the other hand, were all discolored and symptomatic rendering them unmarketable (Fig. 1 panels $B$ and D), though they could attain the marketable size $(15 \mathrm{~cm}$ long and $3.75 \mathrm{~cm}$ in diameter). For size comparison a discolored nontransgenic fruit (arrow) was placed between the transgenic fruits (arrow in Fig. 1 panel C). From Fig. 1 panels $C$ and $D$ it is demonstrated that in the presence of ZYMV and /or WMV, both fruit production and fruit quality of symptomatic transgenic ZW-20 remained unaffected and higher than for nontransgenic cv Pavo.
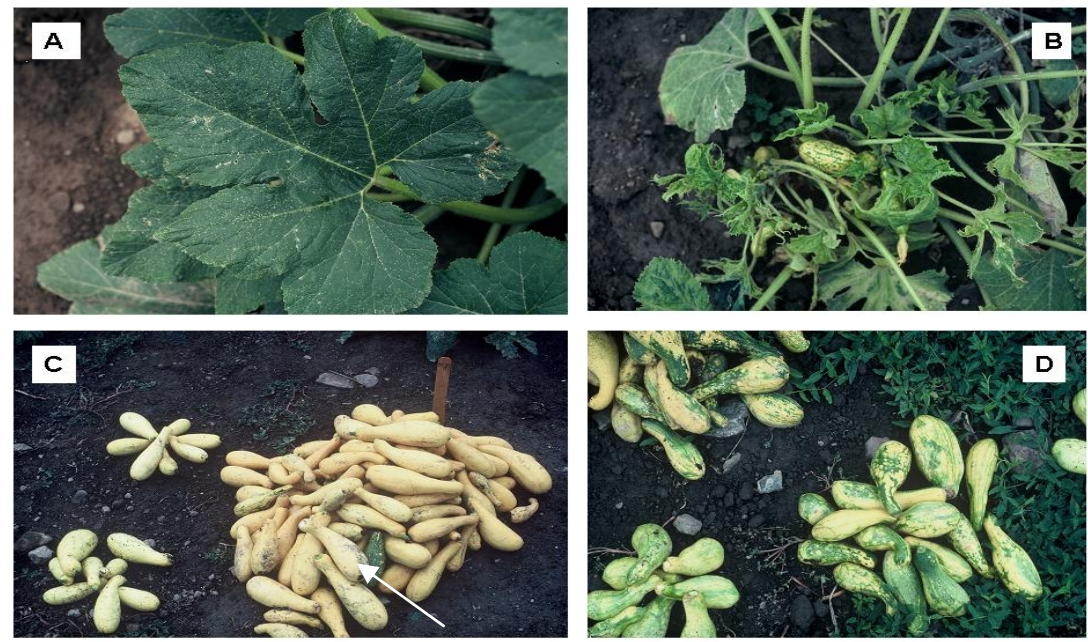

Fig. 1. Comparison of symptoms and fruit productions in transgenic ZW-20 and nontransgenic cv Pavo (control) in the presence of ZYMV and/or WMV.

Panel A: Fully expanded leaves of transgenic $Z W-20 H$, showing some localized chlorotic dots.

Panel B: ZYMV-infected nontransgenic cv Pavo plant expressing severe systemic foliar symptoms consisting of mosaic, leaf deformations and shoestringing, and also bearing a few discolored fruits.

Panel C: Heaps of marketable transgenic $Z W-20 H$ and $B$ fruits. The two small heaps on the left demonstrate fruits of marketable size. The big heap is a combination of slightly oversized fruits and fruits of marketable size. The green discolored fruit (arrow) in this heap is to compare the sizes of marketable transgenic $Z W-20$ fruits and unmarketable nontransgenic cv Pavo fruits.

Panel D: Heaps of virus (both, ZYMV and WMV)-infected, discolored and unmarketable nontransgenic CV Pavo fruits that had reached marketable size. Pictures of panels $A$ and $B$ were taken between 45 and $74 \mathrm{dpp}$. Fruits in panels $C$ and $D$ were photographed after $74 \mathrm{dpp}$, using a portion of all fruits harvested throughout the growing season.

\subsection{Fruit Production Rhythms}

In Fig. 2 (Panel A) the sequence in which the ZW-20B fruits were to reach marketable size and therefore be ready for harvest is indicated by fruit numbering. Fruit number 1 was to be harvested first and fruit number 3 as last one in this series. Additionally, also pistillate flowers occur, which guarantee the continuation of fruit setting, fruit development and harvesting. All this depicts the fruit production rhythm on an unaffected transgenic ZW-20B plant at $45 \mathrm{dpp}$ (mid-season).

In panel B of Fig. 2 the production of low numbers of large oversized matured ZW-20B fruits, harvested at time intervals of 20 - 25 days, is illustrated. 

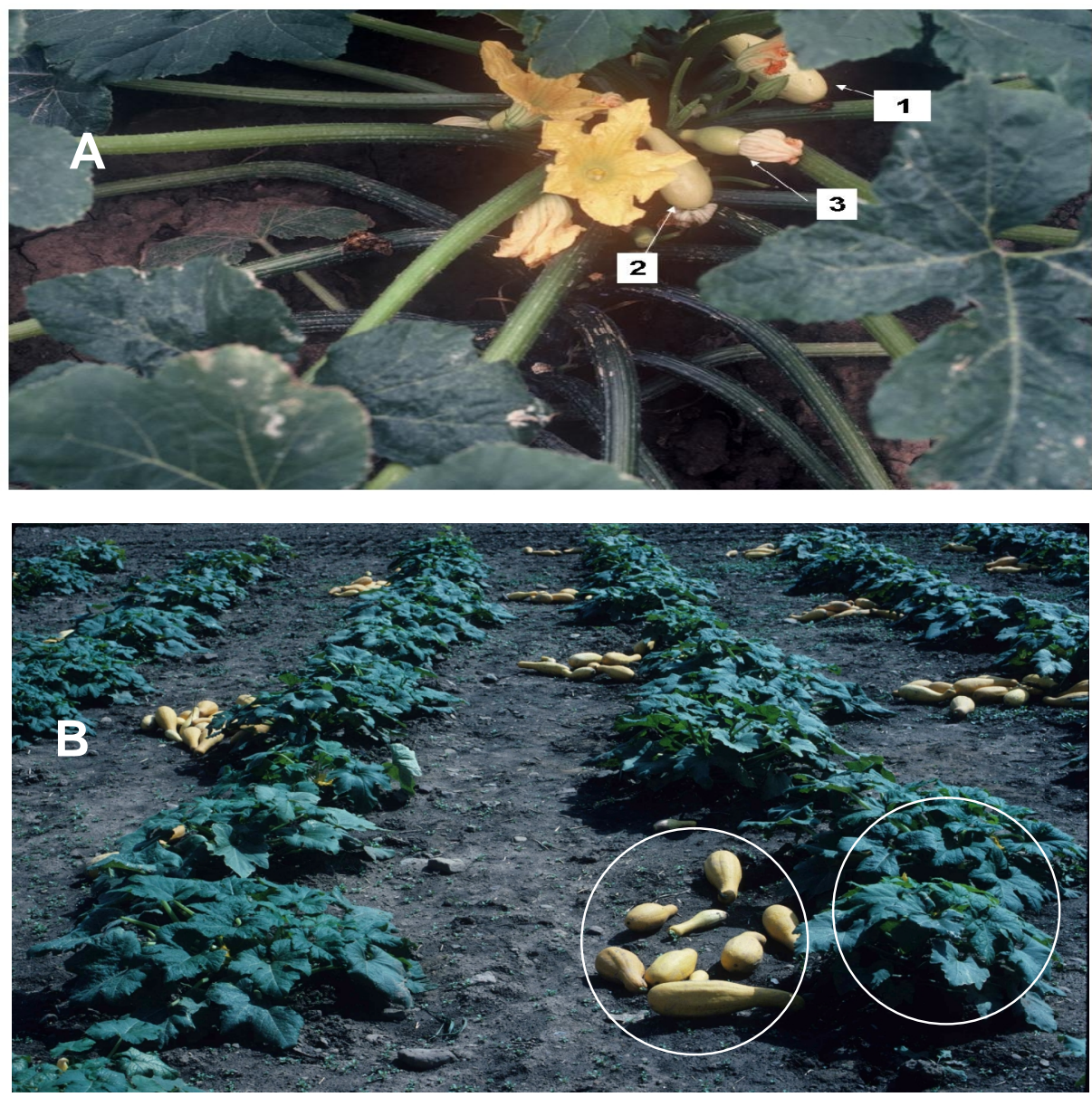

Fig. 2. Panel A. Harvesting young and immature $Z W-20 \bar{B}$ squash of marketable size at a 2-3 day interval. The numbers $(1,2$, and 3$)$ indicate the sequence in which the fruits will reach marketable size and be harvested. Apart from young and immature fruits,

simultaneously also plenty flowers occur to guarantee the continuation of fruit production. All this depicts the fruit production rhythm. Panel B. Harvesting at long time intervals of 20-25 days low numbers of large oversized matured fruits of ZW-20B are obtained. Encircled fruits indicate the low numbers of matured fruits produced by plants 1 and 2 (encircled) altogether over period of 25 days. Panel A was photographed at 45 and panel $B$ at $60 \mathrm{dpp}$

To analyze the impacts ZYMV and WMV could have on the production rhythms, both transgenic ZW-20 and nontransgenic cv Pavo plants were grouped as plants that scored negative in ELISA (NONE) and plants that reacted positively in ELISA (for ZYMV, WMV and ZYMV+WMV). Former group was designated ELISA-negative and latter group ELISApositive. In Fig. 3, time series of average numbers of fruits harvested per plant were plotted against days post planting (dpp) for transgenic genotypes (ZW-20B and ZW-20H) as well as for nontransgenic control cV. Pavo squash, ELISA-positive or - negative (healthy) for ZYMV and WMV. In all panels $(A-H)$ regarding the transgenic genotypes $(\mathrm{ZW}-20 \mathrm{~B}$ and $\mathrm{ZW}-2 \mathrm{OH})$ an 
increasing trend in the production of fruits of marketable size was manifest for successive harvests. This trend generally peaked at 42,45 or $47 \mathrm{dpp}$ and then tapered off towards the end of the growing season. This was true for both, ELISA-negative (NONE) and ELISApositive (ZYMV-, WMV- and ZYMV+WMV - infected) plants of the transgenic ZW-20 genotypes.
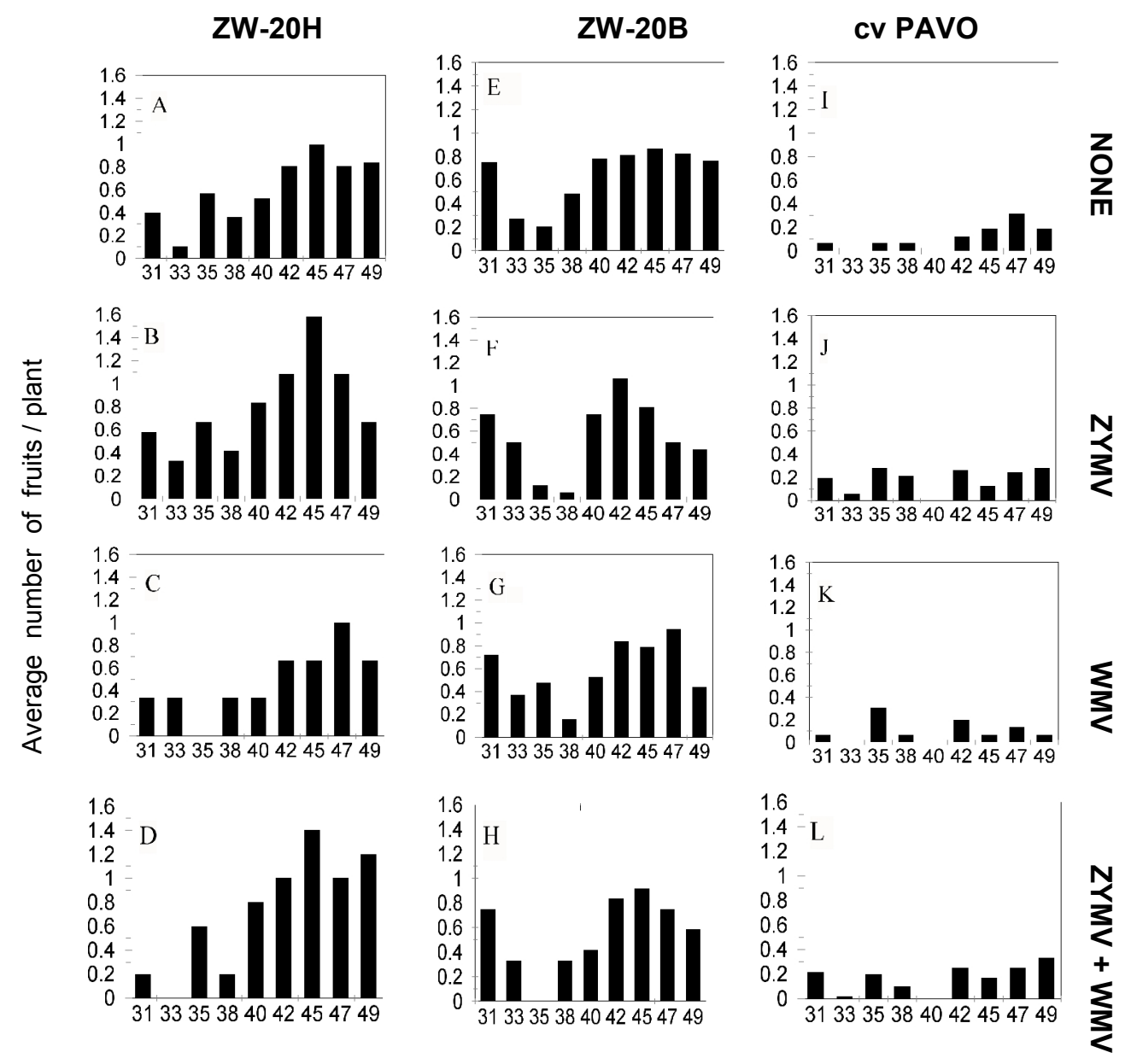

Days post planting

Fig. 3. Graphical representation of the production rhythms of young fruits of marketable size of transgenic ZW-20 ( $H$ and $B$ ) and nontransgenic cv Pavo squash (Cucurbita pepo L), tested ELISA negative or positive for ZYMV, WMV or ZYMV+WMV. Total numbers of fruits $(n)$ and plants $(N)$ per graph can be deduced from Table 1

For nontransgenic cv Pavo squash on the other hand only for ELISA-negative plants an increasing trend of successive production of fruits of marketable size that peaked at $47 \mathrm{dpp}$ was revealed (Fig. 3 panel I). For ELISA-positive (ZYMV-, WMV- and ZYMV+WMV infected) nontransgenic virus susceptible plants, however, no steady increase of the average number fruits of marketable size could be identified. A random pattern appeared in stead (Fig. 3 panels $\mathrm{J}, \mathrm{K}$ and $\mathrm{L}$ ). 


\subsection{Characterization of Fruit Production Rhythms}

Maintaining a left-tail critical region for $Q$ and a critical value of 0.9825 at $P=0.05$ and $m=8$ (from the Handbook of Statistical Tables (Owen, 1962)) Table 1 reveals that all infection groups of transgenic ZW-20 plants, whether ELISA-negative (NONE) or ELISA-positive for ZYMV, WMV and ZYMV + WMV, exhibited significant serial correlations $(Q<0.9825)$ between successive harvests $(\mathrm{ZW}-20 \mathrm{H}: 0.7146,0.9288,0.8318$ and 0.7829 , respectively; and ZW-20B: $0.3610,0.8146,0.7677$ and 0.7005 , respectively).Therefore, indicating the occurrence and retention of autocorrelation in the transgenic genotypes.

Similar to the transgenic crops, only the production rhythm of the group of ELISA-negative plants of the cv Pavo genotype was characterized by a Q-value (0.7384) that was lower than the critical value of 0.9825 ; confirming the existence of autocorrelation in this group. The groups of ELISA-positive (ZYMV, WMV and ZYMV+WMV) cv Pavo plants all had Q-values $(2.4483,1.6282$ and 1.4056 , respectively) that overstepped the critical value of 0.9825 (Table 1) and therefore exhibiting absence of autocorrelation between successive harvests.

Table 1. Characterization of transgenic (ZW-20B and ZW-20H) and non-transgenic squash (cv Pavo) production rhythms by Von Neumann's $Q$

\begin{tabular}{|c|c|c|c|c|c|c|c|c|c|}
\hline \multirow[t]{3}{*}{ Virus } & \multicolumn{9}{|c|}{ Genotype } \\
\hline & \multicolumn{3}{|c|}{$\mathrm{ZW}-20 \mathrm{H}$} & \multicolumn{3}{|c|}{ ZW-20B } & \multicolumn{3}{|c|}{ cv Pavo } \\
\hline & $\mathrm{N}^{\mathrm{a}}$ & $n$ & $\mathbf{Q}^{\mathrm{bc}}$ & $\mathbf{N}$ & $\mathbf{n}$ & $\mathbf{Q}$ & $\mathbf{N}$ & $n$ & $\mathbf{Q}$ \\
\hline NONE $^{d}$ & 124 & 669 & 0.7146 & 92 & 531 & 0.3610 & 7 & 16 & 0.7384 \\
\hline ZYMV & 12 & 87 & 0.9288 & 16 & 78 & 0.8146 & 57 & 94 & 2.4483 \\
\hline $\begin{array}{l}\text { WMV } \\
\text { WMV }\end{array}$ & 3 & 13 & 0.8318 & 19 & 107 & 0.7677 & 15 & 13 & 1.6282 \\
\hline $\begin{array}{c}+ \\
\text { ZYMV }\end{array}$ & 5 & 32 & 0.7829 & 12 & 59 & 0.7005 & 60 & 92 & 1.4056 \\
\hline
\end{tabular}

\subsection{The expanded Von Neumann's Q for the Production Rhythms}

All analysis before were done for adjacent observations $(j=1)$. To further explore the autocorrelation between near-adjacent observations at time lags $j=2,3$ and 4 the expanded Von Neumann's $Q$ was applied. Panels in Fig. 4 present the autocorrelations at these lagvalues for groups of ELISA-negative (NONE) and groups of ELISA-positive (ZYMV-, WMVand $(Z Y M V+W M V))$ transgenic $(Z W-20 B$ and $Z W-20 H)$ on the one hand and nontransgenic cv Pavo (control) squash plants, on the other hand. Values lower than the critical line of $\mathrm{Q}=0.9825$ indicate significant autocorrelation at the respective lag-values, while $Q(j)$-values exceeding the critical line (Fig. 4) manifest the absence of auto-correlation, and therefore the occurrence of randomness between successive observations ( numbers of fruits).

For all three genotypes (resistant transgenic ZW-20 ( $\mathrm{H}$ and $\mathrm{B}$ ) and nontransgenic virussusceptible cv Pavo), significant autocorrelations were found at lag times 1 and 2 for the production rhythms of groups of ELISA-negative (NONE) plants (Fig. 4 panels A, E and I) When ELISA-positive for ZYMV or WMV, autocorrelation in ZW-20 genotypes was primarily 
significant at lag $j=1$. However, when mixed infected with ZYMV and WMV the widest range of lag times showing autocorrelation was revealed between successive harvests for $\mathrm{ZW}-2 \mathrm{OH}$, covering lag $\mathrm{j}=1,2$ and 3 (Fig. 4 panel D).

ZW-20H
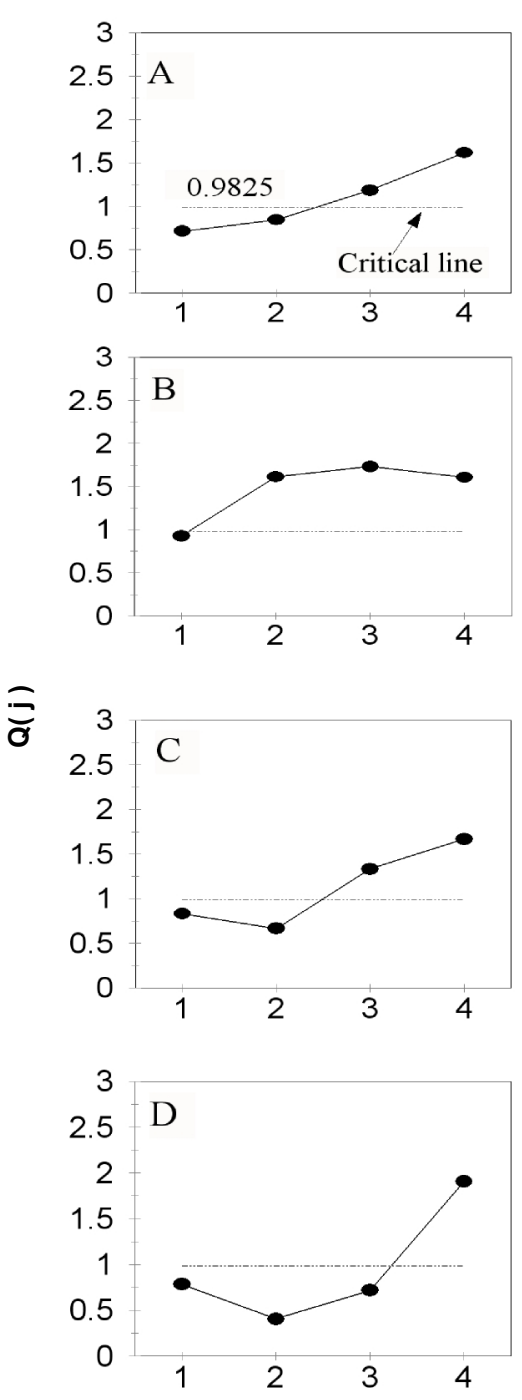

ZW-20B
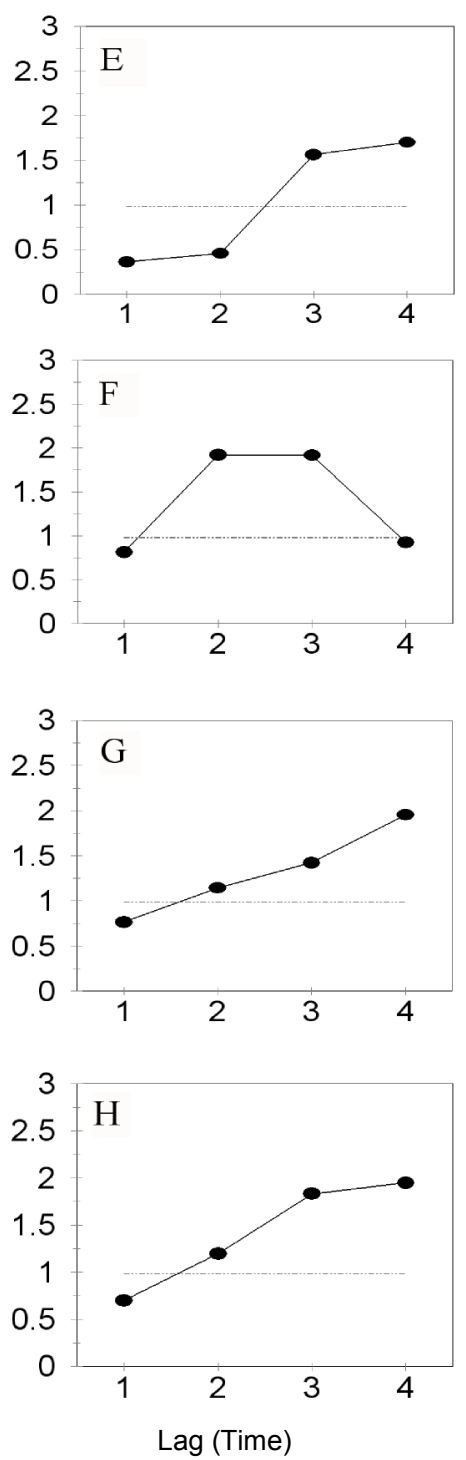

cv PAVO

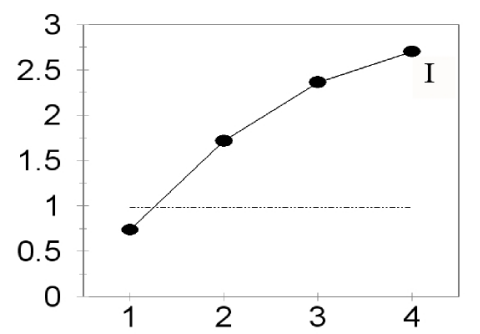

zo

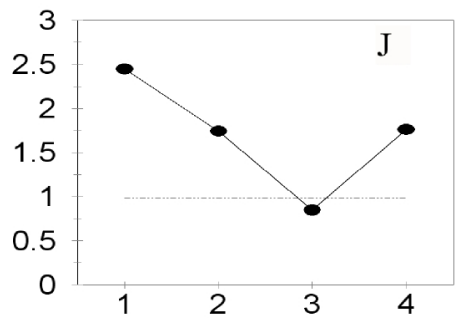

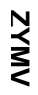

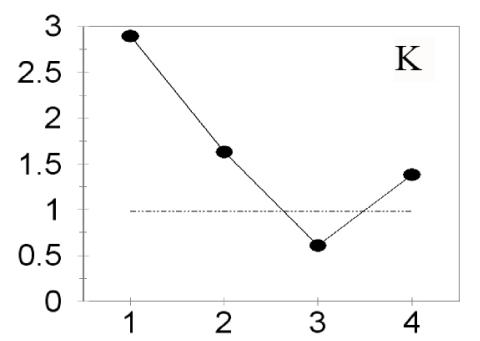

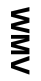

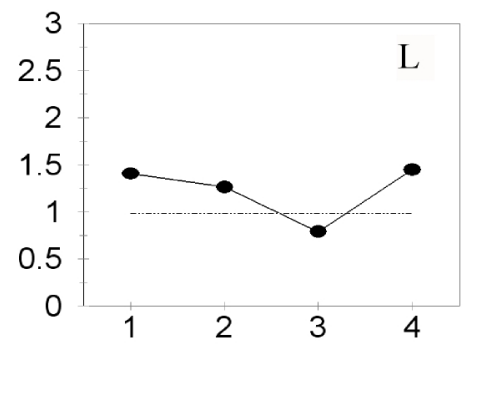

Fig. 4. Expanded Von Neumann's autocorrelation functions at lag $(j)=1,2,3$ and 4 for, both, transgenic ZW - 20 (H and B ) and nontransgenic cV Pavo, reacting negatively or positively for ZYMV, WMV or ZYMV +WMV. Dashed horizontal line represent the left-tail critical value (0.9825) at significance level $\boldsymbol{P}=0.05$ and sample size $\boldsymbol{m}=8$. Values lower than this line are significant and reveal the occurrence of autocorrelation between successive harvests 
In contrast to transgenic ZW-20 (B and $\mathrm{H})$, nontransgenic cv Pavo did not show any significant autocorrelation at $\operatorname{lag} j=1$, when ELISA-positive for any of the viruses (neither single nor mixed infected; but showed this at lag $\mathrm{j}=3$, instead (Fig. 4 panels $\mathrm{J}, \mathrm{K}$ and $\mathrm{L}$ ).

\section{DISCUSSION}

When harvesting fruits in an early and immature stage the resources (photosynthate, mineral nutrients and water) are not employed to enlarging the fruits. Instead, the plant will reallocate the resources for other functions such as growth (additional nodes or side branches) and reproduction (more pistilate flowers). Therefore more small and immature fruits are produced (Charnov, 1982; Watson and Casper, 1984). Avila-Sakar et al. (2001) confirmed that plants (Cucurbita pepo ssp texana) do possess the physiological mechanism to reallocate unused resources. They found that plants that had undergone fruit removal not only grew faster ( produced significantly more nodes) than plants with developing fruits, but also produced a significantly greater proportion of nodes with a flower bud and therefore higher numbers of fruits at successive harvests (Stricker et al., 2008) .

In the present study the phenomenon of an increasing trend in fruit production at successive harvests, was also evident for groups of healthy plants of, both, virus-resistant transgenic ZW-20 and virus-susceptible nontransgenic cv Pavo (Fig. 3 panels A, E and I). Interestingly, the same features were manifest for the production rhythms of groups of transgenic ZW-20 plants infected with ZYMV and / or WMV (Fig. 3 panels B, C, D, F, G and H) and reacting positively in ELISA.

In contrast, production rhythms of nontransgenic virus-susceptible cv Pavo, infected with ZYMV and/or WMV (positive ELISA reaction), lacked the increasing trend in fruit production at successive harvests (Fig. 3 panels $\mathrm{J}, \mathrm{K}$ and $\mathrm{L}$ ).

It is remarkable that yields of healthy nontransgenic cv Pavo plants were lower (Fig. 3 panel I) than of healthy ZW-20 squash (Fig. 3 panels A and E). An explanation to this discrepancy is the following: ELISA tests were performed at 14, 45 and $74 \mathrm{dpp}$. Seven of the nontransgenic cv Pavo plants that were healthy and reacting negatively at $14 \mathrm{dpp}$ retained that status at $74 \mathrm{dpp}$ (end of season) and yielded only 16 fruits of marketable size on the successive harvest dates. However, despite this low number of fruits, the successive harvests of these 16 fruits from healthy $\mathrm{cv}$ Pavo plants showed an increasing trend (Fig. 3 panel I) and were significantly autocorrelated at Lag $\mathrm{j}=1$ (Table 1). This indicates that the occurrence or absence of an increasing trend in successive harvests in more decisive for the occurrence or absence of autocorrelation within the production rhythms, than low or high numbers of plants and fruits within an infection group.

Characterization by Von Neumann's $Q$ revealed that autocorrelation in the production rhythms of ZW-20 was retained, irrespective if the plants were virus-infected (ZYMV or WMV) or not. On the other hand Von Neumann's Q provided the evidence that loss of autocorrelation between successive fruit productions of cv Pavo was strongly associated with ZYMV and WMV infections. These outcomes sustain the hypothesis that the cucurbit viruses ZYMV and WMV have no impact on the production rhythms of ZW-20 squash, but they do have an impact on that of nontransgenic cv Pavo.

Autocorrelation analysis is a commonly-used tool for checking non-randomness (autocorrelation) in a set of data (Von Neumann, 1941). In this respect it is usually the lag $\mathrm{j}=$ 1 autocorrelation that is of interest. 
However, to study the behavior of autocorrelation at higher time lags, Von Neumann's $Q$ was expanded (equation 4) to lags $\mathrm{j}=2,3$ and 4 . In this respect $Z \mathrm{~W}-20 \mathrm{H}$ exhibited a slightly better performance than ZW-20B; in that, WMV-infected ZW-20H (Fig. 4. panel C) had a wider range (lag $\mathrm{j}=1$ and 2) of autocorrelation than similarly infected ZW-20B (Fig. 4. panel G: autocorrelation only lag $j=1)$. This range was even wider for mixed $(Z Y M V+W M V)$ infected $\mathrm{ZW}-20 \mathrm{H}$ (Fig. 4. panel D: autocorrelation at lag $\mathrm{j}=1,2$ and 3) as compared to ZW-20B (Fig. 4 panel $\mathrm{H}$ : autocorrelation only at lag $\mathrm{j}=1$ ).

This finding indicates that $\mathrm{ZW}-20 \mathrm{H}$ has a higher potential to maintain its production rhythm than ZW-20B under conditions of high infection pressures of ZYMV and/ or WMV. In an earlier publication (Klas et al., 2006) the slightly better performance of ZW-20H over ZW-20B also became manifest as latter genotype showed some potential to serve as a source for secondary vector transmission of ZYMV and WMV, while in former genotype such potential was absolutely absent. Despite these differences between the transgenic genotypes fruit quality and yield were similarly high (Totals of the $n$ columns in Table 1 and Klas et al., 2011).

On the other hand Fig. 4 (panels $I, J, K$ and $L$ ) shows a shift of autocorrelation from lag $j=1$ (for healthy cv Pavo) to lag j = 3 (for ZYMV-, WMV- and (ZYMV + WMV) - Infected cv Pavo). This virtually implies the disappearance of autocorrelation between (adjacent) successive harvests of cv Pavo fruits of marketable size, and therefore exhibits the impact of these viruses on the production rhythm of nontransgenic cv Pavo.

There are several documents reporting upon periodic patterns in the seasonal production of summer squash (e.g. Stricker et al., 2008). However, none of these publications have addressed the issue of characterization and quantification of these patterns by a statistic. In this work, squash fruit production rhythms were characterized by Von Neumann's Q. The outcomes of this statistic were then used to evaluate the impacts of ZYMV and WMV on production rhythms of transgenic and nontransgenic squash. To the best of my knowledge, such has not been done before.

\section{CONCLUSION}

Most studies on disease impact on a crop pertain to the extent of yield reductions caused, and do not consider the impact a disease can have on the production rhythm. Therefore in this work, a first impulse is given to this approach. The main findings achieved, and conclusions arrived at are:

1. Evidence is provided that the cucurbit viruses ZYMV and WMV have a destructive impact the production rhythm of non transgenic cv Pavo squash.

2. Cucurbit viruses ZYMV and WMV have not shown any impact on transgenic ZW$20 \mathrm{H}$ and $\mathrm{ZW}-20 \mathrm{~B}$ squash genotypes, which are resistant, but not immune to these viruses.

3. Infected transgenic $Z \mathrm{~W}-20 \mathrm{H}$ has shown autocorrelation over a wider range of lag times than infected $Z W-20 B$. This finding predicts a higher potential for the former genotype to retain its production rhythm under conditions of high infection pressures of ZYMV and WMV.

4. In terms of farming practices these results imply that even in the presence of high ZYMV and WMV infection pressures, the demanded volumes of fruits of marketable size can be delivered timely (daily) to the market when growing transgenic virus-resistant ZW-20 squash. In contrast, the availability of fruits of 
marketable size (but unmarketable quality) from infected nontransgenic cv Pavo plants, will be substantially delayed.

\section{ACKNOWLEDGEMENTS}

This work was done at the Department of Plant Pathology and Plant-Microbe Biology of Cornell University Geneva, NY, 14456. Thanks are due Dennis Gonsalves for my participation in his USDA Biotechnology Risk Assessment Grant Program (No 95-331201878) and for the instructive discussions.

\section{COMPETING INTERESTS}

Author has declared that no competing interests exist.

\section{REFERENCES}

Agrawal, A.A. (1999). Induced responses to herbivory in wild radish: effects on several herbivores and plant fitness. Ecology, 80, 1713-1723.

Avila-Sakar, G.G., Krupnick, G.A., Stephenson, A.G. (2001). Growth and resource allocation in Cucurbita pepo spp texana: Effects af fruit removal. Int. J. Plant Sci., 162(5), 1089 1095.

Bazzaz, F.A. (1997). Allocation of resources in plants: state of the science and critical questions. Pages 1-38 in F.A. Bazzaz, J. Grace, eds. Plant Resource Allocation. Academic Press, San Diego, Calif.

Buishand, T.A. (1982). Some methods for testing the homogeneity of rainfall records. Journal of Hydrology, 58, 11-27.

Charlesworth, D., Charlesworth, B. (1982). Allocation of resources to male and female functions in hermaphrodites. Biol. J. Linn Soc, 15, 57-74.

Charnov, E.L. (1982). The theory of sex allocation. Princeton University Press, Princeton, N.J., 355.

Cipolini, D.F. (1999). Costs to flowering of the production of a mechanically hardened phenotype in Brassica napus L. Int J. Plant Sci., 160, 735-741.

Cipolini, D.F., Schultz, J.C. (1999). Exploring the cost constraints on stem elongation in plant using phenotypic manipulation. Am Nat, 153, 236-242.

Dudley, S.A., Schmitt, J. (1995). Genetic differentiatin in morphological responses to simulated foliage shade between populations on Impatiens capensis from open woodland sites. Funct Ecol, 9, 655-666.

Delesalle, V.A., Mooreside, P.D. (1995). Estimating the costs of allocation to male and female functions in a monoecious cucurbit, Lagenaria siceraria. Oecologia, 102, 9-16.

El-Keblawy, A., Louvett-Doust, J. (1996). Resource re-allocation following fruit removal in cucurbits: patterns in cantaloupe melons. New Phytol., 134, 413-422.

Fuchs, M., Tricoli, D.M., Carney, K.J., Schlesser, M., McFerson, J., Gonsalves, D. (1998). Comparative virus resistance and fruit yield of transgenic squash with single and multiple coat protein genes. Plant Disease, 82, 1350-1356.

Klas, F.E., Fuchs, M., Gonsalves, D. (2006). Comparative spatial spread overtime of zucchini yellow mosaic virus (ZYMV) and watermelon mosaic virus (WMV) in fields of transgenic squash expressing the coat protein genes of ZYMV and WMV and in fields of nontransgenic squash. Transgenic Research, 15, 527-541. 
Klas, F.E., Fuchs, M., Gonsalves, D. (2011). Field yield of virus-resistant transgenic summer squash in simulated commercial plantings under conditions of high disease pressure. Journal of Horticulture and Forestry, 3(2), February 2011, 46-52.

Krupnick, G.A., Brown, K., Stephenson, A.G. (1999). Influence of fruit on the regulation of internal ethylene concentrations and sex expressin in Cucurbita texana. Int J Plant Sci 160, 321-330.

Schlichting, C.D., Pigliucci, M. (1998). Phenotypic evolution: a reaction norm perspective. Sinauer, Sunderland, Mass, 387.

Owen, D.B. (1962). Handbook of statistical tables. reading mass. Addison-Wesley Pub. Co.

Schmitt, J., Dudley, S.A., Pigliucci, M. (1999). Manipulative approaches to testing adaptive plasticity: phytochrome-mediated shade-avoidance responses in plants. Am Nat, 154, 43-54.

Schmitt, J., McCormac, A.C., Smith, H. (1995). A test of the adaptive plasticity hypothesis using transgenic and mutant plants disabled in phytochrome-mediated elongation responses to neighbors. Am Nat, 146, 937-953.

Strickler, K., Laughlin, K., Agenbroad, A. (2008). Harves frequency, yield and economics of summer squash. Final report of western sustainable agricultural research and education program. Project Number FW06-042. http:// www.pollinatorparadise.com/Squash_Project/SquashResearch.htm.

Tricoli, D., Carney, K.J., Russell, P.F., McMaster, J.R., Groff, D.W., Hadden, K.C., Himmel, P.T., Hubbard, J.P., Boeshore, M.L., Quemada, H.D. (1995). Field evaluation of transgenic squash containing single or multiple virus coat protein gene constructs for resistance to cucumber mosaic virus, watermelon mosaic virus 2 and zucchini yellow mosaic virus. Bio/Technol., 13, 1458-1465.

Von Neumann, J. (1941). Distribution of the ratio of the mean square successive difference to the variance. The Annals of Mathematical Statistics, 12(4), 367-395.

Wang, Y. (2003). Nonparametric tests for randomness. ECE 461 Project Report.

Wardlaw, I.F. (1990). The control of carbon partioning in plants. New Phytol, 116, 341-381.

Watson, M.A., Casper, B.B. (1984). Morphogenetic constraints on patterns of carbon in plants. Annu Rev Ecol Syst, 15, 223-258.

Yampolski, C., Yampolski, H. (1922). Distribution of sex forms in the phanerogamic flora. Bibl Genet, 3, 1- 62. 


\section{APPENDIX}

Von Neumann's $Q$ is presented as in equation (1) as:

$$
Q=\frac{\sum_{i=1}^{m-1}\left(x_{i+1}-x_{i}\right)^{2}}{\sum_{i=1}^{m}\left(x_{i}-\bar{x}\right)^{2}}
$$

By dividing both, the numerator and denominator of $Q$ by $\mathrm{m}-1$ we find for the numerator:

$$
\frac{\sum_{i=1}^{m-1}\left(x_{i+1}-x_{i}\right)^{2}}{m-1}
$$

which equals $\varepsilon\left(x_{i+1}-x_{i}\right)^{2}$, the expectation of the squared difference between successive measurements.

We know that $\varepsilon\left(x_{i+1}\right)=\varepsilon\left(x_{i}\right)$, therefore $\varepsilon\left(x_{i+1}-x_{i}\right)=0$.

Consequently we can re-write equation (2) as:

$$
\frac{\sum_{i=1}^{m-1}\left(x_{i+1}-x_{i}\right)^{2}}{m-1}=\varepsilon\left(x_{i+1}-x_{i}\right)^{2}-\left\{\varepsilon\left(x_{i+1}-x_{i}\right)\right\}^{2}=\operatorname{var}\left(x_{i+1}-x_{i}\right)
$$

Furthermore we know that

$$
\begin{aligned}
& \operatorname{var}\left(x_{i+1}-x_{i}\right)=\operatorname{var} x_{i+1}+\operatorname{var} x_{i}-2 \operatorname{cov}\left(x_{i+1}, x_{i}\right) \text { or } \\
& \operatorname{var}\left(x_{i+1}-x_{i}\right)=\operatorname{var} x_{i+1}+\operatorname{var} x_{i}-\frac{2 \operatorname{cov}\left(x_{i+1}, x_{i}\right)}{\sqrt{\operatorname{var} x_{i+1}} \sqrt{\operatorname{var} x_{i}}} \sqrt{\operatorname{var} x_{i+1}} \sqrt{\operatorname{var} x_{i}} \\
& \operatorname{var} x_{i+1}=\operatorname{var} x_{i}=\sigma^{2} \\
& \text { Therefore, } \operatorname{var}\left(x_{i+1}-x_{i}\right)=2 \sigma^{2}-2 \rho \sigma^{2}=2 \sigma^{2}(1-\rho)
\end{aligned}
$$

From (3) and (4) we find that the numerator of $Q$ equals:

$$
\frac{\sum_{i=1}^{m-1}\left(x_{i+1}-x_{i}\right)^{2}}{m-1}=2 \sigma^{2}(1-\rho)
$$

Division of the denominator of $Q$ by $m-1$ yields the sample variance, 
and we find,

$$
\frac{\sum_{i=1}^{m-1}\left(x_{i}-\bar{x}\right)^{2}}{m-1}=S^{2}
$$

$$
Q=\frac{2 \sigma^{2}(1-\rho)}{S^{2}}
$$

Furthermore, $S^{2} \rightarrow \sigma^{2} \quad$ as $\quad(m-1) \rightarrow \infty$

$$
\text { Therefore, } Q=2(1-\rho) \text {. }
$$

(C) 2012 Klas; This is an Open Access article distributed under the terms of the Creative Commons Attribution License (http://creativecommons.org/licenses/by/3.0), which permits unrestricted use, distribution, and reproduction in any medium, provided the original work is properly cited. 